\title{
Management of Unilateral Vocal Cord Palsy: Case Series with Review of Literature
}

\author{
1Priyanka J Hardikar, ${ }^{2}$ Jyoti P Dabholkar, ${ }^{3}$ Ramprasath Vasan, ${ }^{4}$ Nilam Sathe
}

\begin{abstract}
Aim: To find out the vocal outcome of conservative management and temporary medialization in unilateral vocal cord palsy (UVCP) and their effectiveness in reducing the need for permanent medialization.
\end{abstract}

Materials and methods: Thirty adult patients with UVCP, without structural vocal cord lesion or laryngeal malignancy were initially treated conservatively, and those without adequate response at 3 months were treated with temporary medialization. They were followed up for 6 months. Pre- and posttreatment voice was compared using grade, roughness, breathiness, asthenia, strain (GRBAS) scale, Voice Handicap Index (VHI), and maximum phonation time (MPT).

Results: The results show $40 \%$ cases were idiopathic, whereas $33.33 \%$ had iatrogenic cause, majority, following thyroidectomy; $66.67 \%$ responded well to the conservative management. Ten patients (33.33\%) did not show satisfactory improvement in the first 3 months and were treated with temporary medialization. Both the groups were comparable at the first visit. At 6 months, the improvement in all parameters was significantly more in the temporary medialization group than the conservative management group compared with their values at 3 months. Two of the 10 patients treated with injection laryngoplasty were considered for permanent medialization. The final outcome was similar in both the groups.

Conclusion: Conservative management of UVCP is the standard of care. Injection laryngoplasty at 3 months achieves better voice outcome in those not fully improved by conservative management. Medialization thyroplasty should be suggested in patients failing to show the desired improvement after injection laryngoplasty.

Clinical significance: The study guides in the management of UVCP, in terms of its likely cause and approach to treatment.

Keywords: Conservative management, Injection laryngoplasty, Prospective study, Unilateral vocal cord palsy.

\footnotetext{
${ }^{1}$ Senior Resident, ${ }^{2}$ Professor Emeritus, ${ }^{3}$ Junior Resident ${ }^{4}$ Associate Professor

${ }^{1-4}$ Department of ENT, Seth Gordhandas Sunderdas Medical College and King Edward Memorial Hospital, Mumbai Maharashtra, India

Corresponding Author: Priyanka J Hardikar, Senior Resident, Department of ENT, Seth Gordhandas Sunderdas Medical College and King Edward Memorial Hospital Mumbai, Maharashtra, India, Phone: +912224156611, e-mail: priyankahardikar10@gmail.com
}

How to cite this article: Hardikar PJ, Dabholkar JP, Vasan R, Sathe N. Management of Unilateral Vocal Cord Palsy: Case Series with Review of Literature. Int J Phonosurg Laryngol 2017;7(2):52-58.

\section{Source of support: Nil}

\section{Conflict of interest: None}

\section{INTRODUCTION}

The human voice serves a great deal of communicative function and is the best expression of emotions. Voice change can have a lot of functional, emotional, psychological, and professional impacts on a person's life.

Unilateral vocal cord palsy is a commonly encountered condition and these patients can be asymptomatic in one-third of the cases or present with dysphonia, aspiration, vocal fatigue, and rarely potential respiratory compromise. In addition to the disruption of the physiological laryngeal function, it can also interfere with the quality of life, especially in professionals for whom voice quality is important.

Vocal cord movement is brought about by the intrinsic laryngeal muscles, innervated mainly by the recurrent laryngeal nerve (RLN) except the cricothyroid muscle which is innervated by the external branch of the superior laryngeal nerve, both being branches of the vagus nerve. Vocal cord palsy refers to vocal cord immobility and can result from central nervous system disorders, vagal nuclear or supranuclear lesions, vagus nerve lesions itself, or RLN lesions. If the nerve injury is transient, the patients experience complete recovery of the vocal cord function. However, if the injury is severe, and complete conduction block develops, the patient may not regain appropriate vocal fold movement, in spite of signs of reinnervation on electromyography. Recovery may be complete or incomplete with compensation. The effect on voice can be evaluated by perceptual assessment like the GRBAS scale and Consensus Auditory Perceptual Evaluation-Voice (CAPE-V) scales, subjective evaluation in the form of VHI as well as assessment of aerodynamic parameters like MPT.

The treatment is initially conservative including speech therapy, pushing exercises, swallowing therapy, and electrical stimulation. Temporary medialization using injection laryngoplasty can be done before attempting a permanent vocal cord medialization with Type I 
medialization thyroplasty. Arytenoid adduction can be used to supplement a Type I thyroplasty if the posterior glottic gap is large. Laryngeal reinnervation procedures are designed to bypass the main trunk of the nerve and address the individual intrinsic laryngeal muscles directly or at the most distal branches of the RLN to provide improvement in laryngeal muscle tone and mass.

The aim of this study was to find the vocal outcome of conservative management of UVCP and temporary medialization procedures and the eventual need for a permanent medialization thyroplasty.

\section{MATERIALS AND METHODS}

The study was conducted as a prospective study in the Department of ENT of our tertiary care hospital. A total of 30 adult patients presenting with change in voice, vocal fatigue, aspiration or respiratory distress, and having $\mathrm{UVCP}$ on $70^{\circ}$ rigid endoscopy were enrolled in our study, after ruling out any structural lesion of the vocal cords or laryngeal malignancy. A high-resolution computed tomography (CT) scan from the skull base to mediastinum was done to find out the etiology, along with detailed history and clinical examination. The patients without any relevant history of neck, skull base or thoracic surgery, or prior intubation with a normal CT scan were labeled to have an idiopathic vocal cord palsy.

Auditory perceptual evaluation of voice was done using the GRBAS scale with five parameters: Overall grade or severity $(G)$, roughness of the voice $(R)$, breathiness (B), asthenia (A), and strain (S). The quality of the voice was then given a scoring of 0 to 3 for each parameter such that $0=$ normal, $1=$ mild, $2=$ moderate, $3=$ severe. An overall GRBAS score was obtained.

Aerodynamic voice assessment was done using the MPT. The patient was asked to take a deep breath and sustain the vowel sound "a" for as long as possible at a comfortable pitch and loudness on one exhalation, and without straining. The time was recorded in seconds, and MPT was calculated as the average of three attempts.

Voice Handicap Index was used to assess the subjective feeling of handicap that a patient experiences due to his/her voice problem. The patients were given a questionnaire containing 30 questions of three categories: Functional, physical, and emotional with 10 questions in each category and were asked to give their responses using a score from 0 to 4 such that $0=$ never, $1=$ almost never, 2 = sometimes, 3 = almost always, and $4=$ always . The total score was used as the VHI.

All the patients were initially treated with speech therapy, swallowing therapy, instructed about vocal hygiene, and started on proton pump inhibitors. Patients with tuberculosis were started on anti-tuberculosis treatment and those with other infections like skull base osteomyelitis were started on appropriate antibiotics. Patients were asked to follow-up at 3 months after their first visit, and GRBAS scoring, VHI scoring, and calculation of the MPT were done at the first visit as well as follow-up.

At 3 months, the patients showing significant improvement, i.e., reduction in the GRBAS score of $\geq 5$, increase in the MPT of $\geq 5$ seconds, and reduction in the $\mathrm{VHI} \geq 20$ were continued on a conservative management, whereas patients failing to show improvement were then treated with temporary medialization using injection laryngoplasty with hyaluronic acid-Esthelis Basic (produced by Merz Pharmaceuticals, distributed in India by Aakaar Medical Technologies Pvt Ltd, formerly Coherent Medical Systems). Suspension microlaryngoscopy under general anesthesia was used for the procedure and 0.5 to $1 \mathrm{~mL}$ of hyaluronic acid was injected in the vibratory membrane to achieve the desired cord medialization. All the patients were further followed up for 3 months and at the end of total 6 months from the first visit, the patients were reassessed. The results were computed based on the voice outcome following the two modalities of treatment. The parameters of GRBAS and VHI were analyzed using the Wilcoxon signed rank test in the same group and the Mann-Whitney U-test for the comparison between the two groups of patients. The MPT was analyzed using Student's t-test. An assessment was also made regarding the need of a permanent medialization thyroplasty in patients with a poor voice outcome.

\section{RESULTS}

Of the 30 patients, 18 (60\%) were males and $12(40 \%)$ were females, the youngest being 19 years of age and the oldest being 68 years of age. The most common age group was 41 to 50 years. Eleven (36.67\%) had right cord palsy, whereas 19 (63.33\%) had left cord palsy. All the patients presented with hoarseness or change in voice. In addition to this, the most common symptom was vocal fatigue followed by aspiration. Twelve (40\%) patients had idiopathic palsy. Postsurgical causes accounted for 33.33\% (10) of the patients, among which 7 cases (23.33\%) were postthyroidectomy. The other three cases were: coronary artery bypass graft (CABG), vagal paraganglioma, and vagal schwannoma excision. The other causes included tuberculous mediastinal lymphadenopathy in 4 (13.33\%) patients, pulmonary tuberculosis, skull base osteomyelitis, skull base hyperostosis, and post traumatic in 1 (3.33\%) patient each (Graph 1). At the end of 3 months, two groups were formed: the first of 20 patients who were continued on conservative management and the other of remaining 10 patients who were treated with injection laryngoplasty. Out of these 10 patients, 2 did not improve even after the 


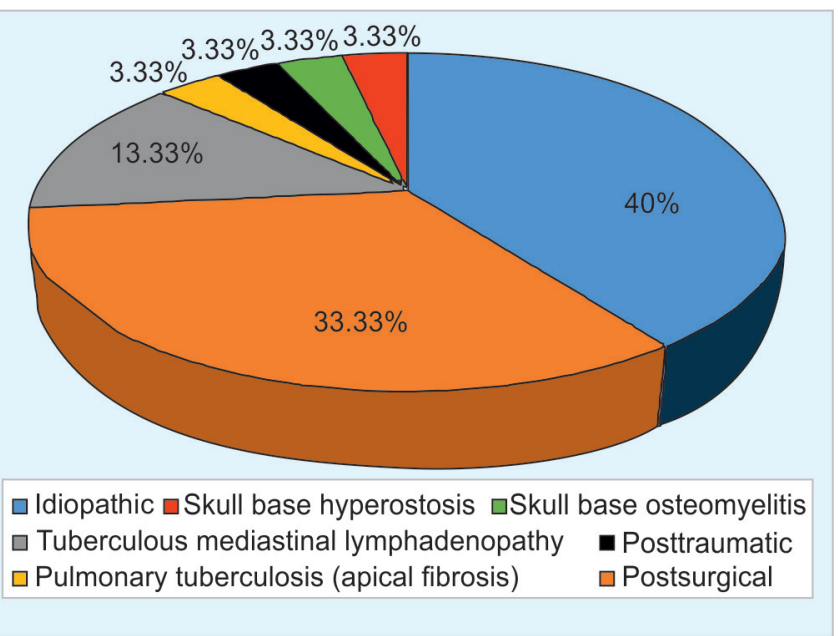

Graph 1: Etiology of unilateral vocal cord palsy in our study

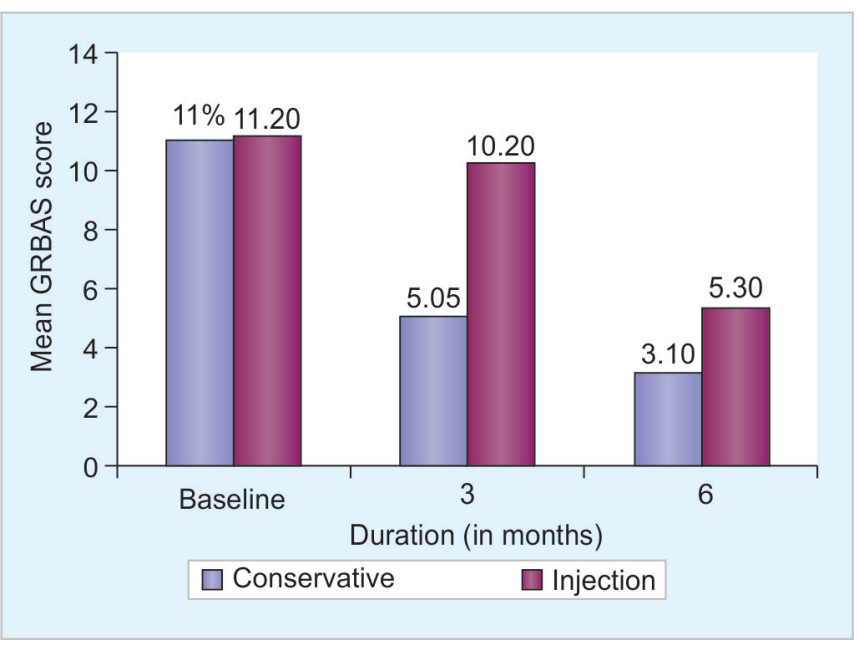

Graph 2: Comparison of changes in mean GRBAS score between the groups

Table 1: Changes in the mean GRBAS score between the groups

\begin{tabular}{|c|c|c|c|}
\hline \multirow[b]{2}{*}{ Duration (months) } & \multicolumn{2}{|c|}{ Mean GRBAS score $(\bar{X} \pm S D)$} & \multirow[b]{2}{*}{$p$-value } \\
\hline & $\begin{array}{l}\text { Conservative management } \\
(n=20)\end{array}$ & $\begin{array}{l}\text { Temporary medialization (injection } \\
\text { laryngoplasty) }(n=10)\end{array}$ & \\
\hline Baseline & $11.00 \pm 01.03$ & $11.20 \pm 01.69$ & $0.734 \mathrm{NS}$ \\
\hline 3 & $05.05 \pm 00.51$ & $10.20 \pm 01.40$ & \\
\hline 6 & $03.10 \pm 00.85$ & $05.30 \pm 02.63$ & \\
\hline Diff $(3-6 m) p$-value & ${ }^{a}-01.95 \pm 00.69(0.001)$ & $a_{-}-04.90 \pm 02.81(0.001)$ & ${ }^{\mathrm{a}} 0.002$ \\
\hline Diff (Baseline $-3 \mathrm{~m}$ ) p-value & ${ }^{a}-05.95 \pm 00.89(0.001)$ & ${ }^{a}-01.00 \pm 00.47(0.001)$ & ${ }^{\mathrm{a}} 0.001$ \\
\hline Diff (Baseline $-6 \mathrm{~m}$ ) p-value & ${ }^{a}-07.90 \pm 01.12(0.001)$ & ${ }^{a}-05.90 \pm 03.00(0.001)$ & $0.051 \mathrm{NS}$ \\
\hline
\end{tabular}

Wilcoxon signed rank test (within group); SD: Standard deviation; NS: Not significant; by Mann-Whitney U-test (between groups); aSignificant

temporary medialization procedure and were considered for permanent medialization by Type I thyroplasty. Both the groups were retrospectively assessed and compared from the baseline values.

\section{Perceptual Evaluation}

Baseline GRBAS score was comparable between the two groups (Graph 2 and Table 1). After 3 months, GRBAS score showed a significant fall of $54.1 \%(\mathrm{p}<0.05)$ in the conservative group and $8.9 \%$ in the temporary medialization group. After a total of 6 months, fall of $38.6 \%$ from the values at 3 months was observed in conservative management group as against $48.0 \%$ in temporary medialization group. The GRBAS scores showed a significant fall of $71.8 \%$ within the conservative treatment group and $52.7 \%$ within the temporary medialization group from the baseline. The difference between two groups at the end of 6 months was not significant.

\section{Aerodynamic Analysis}

Baseline MPT showed no significant difference between the two groups (Graph 3 and Table 2). At the end of 3 months, a significant rise $(\mathrm{p}<0.05)$ of 1.30 times in the

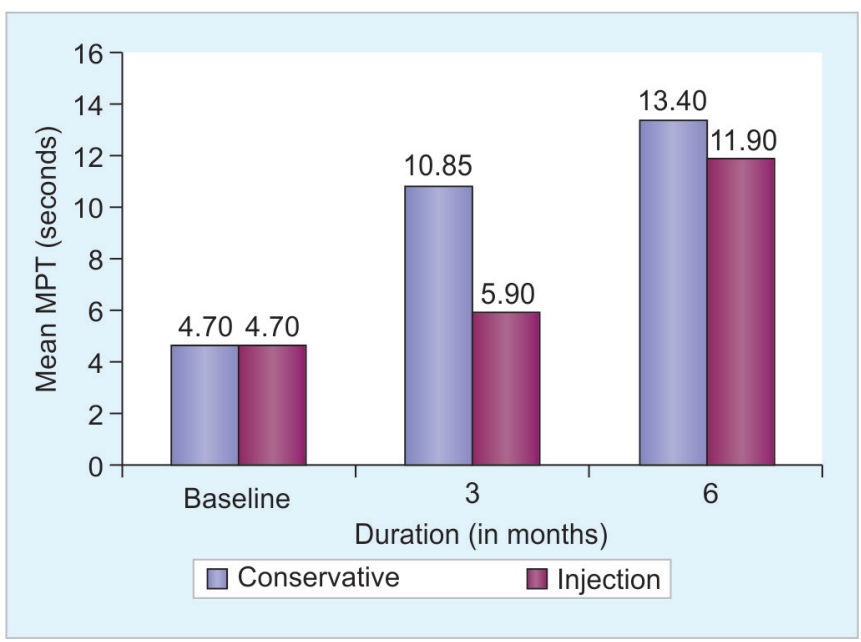

Graph 3: Comparison of changes in mean MPT between the groups

conservative group and $25.5 \%$ in the temporary medialization group was observed, compared with the baseline; significantly more $(\mathrm{p}<0.05)$ in the conservative treatment group. At 6 months, a rise of $23.5 \%$ in the conservative group and 1.01 times in temporary medialization group from the respective values at 3 months was observed, the rise being significantly more $(p<0.05)$ in the temporary 
Table 2: Changes in the mean MPT between the groups

\begin{tabular}{llll}
\hline & \multicolumn{2}{c}{ Mean MPT (seconds) $(\bar{X} \pm S D)$} \\
\cline { 2 - 3 } Duration (months) & $\begin{array}{l}\text { Conservative management } \\
(n=20)\end{array}$ & $\begin{array}{l}\text { Temporary medialization (injection } \\
\text { laryngoplasty) }(n=10)\end{array}$ & $p$-value \\
\hline Baseline & $04.70 \pm 00.80$ & $04.70 \pm 00.82$ & $1.000 \mathrm{NS}$ \\
3 & $10.85 \pm 00.81$ & $05.90 \pm 01.10$ & \\
6 & $13.40 \pm 00.94$ & $11.90 \pm 02.51$ & ${ }^{\mathrm{a}} 0.001$ \\
Diff $(3-6 \mathrm{~m})$ p-value & ${ }^{\mathrm{a}} 02.55 \pm 00.51(0.001)$ & ${ }^{\mathrm{a}} 06.00 \pm 02.16(0.001)$ & ${ }^{\mathrm{a}} 0.001$ \\
Diff (Baseline $-3 \mathrm{~m})$ p-value & ${ }^{\mathrm{a}} 06.15 \pm 00.59(0.001)$ & ${ }^{\mathrm{a}} 01.20 \pm 00.42(0.001)$ & ${ }^{\mathrm{a}} 0.045$ \\
Diff (Baseline $-6 \mathrm{~m}) \mathrm{p}$-value & ${ }^{\mathrm{a}} 08.70 \pm 00.80(0.001)$ & ${ }^{\mathrm{a}} 07.20 \pm 02.20(0.001)$ & \\
\hline
\end{tabular}

Student's t-test; SD: Standard deviation; NS: Not significant; ${ }^{\text {a Significant }}$

medialization group. Thus, at the end of 6 months, a significant rise in the MPT, of 1.85 times in conservative group and 1.53 times in temporary medialization group, was observed from baseline $(\mathrm{p}<0.05)$.

\section{Subjective Analysis}

Baseline VHI did not show a significant difference in both the groups (Graph 4 and Table 3). After 3 months, there was a significant fall $(\mathrm{p}<0.05)$ of $29.1 \%$ in the conservative group and $2.6 \%$ in the temporary medialization group; significantly more $(\mathrm{p}<0.05)$ in the conservative treatment group. After 6 months, a significant fall $(p<0.05)$ of $21.8 \%$

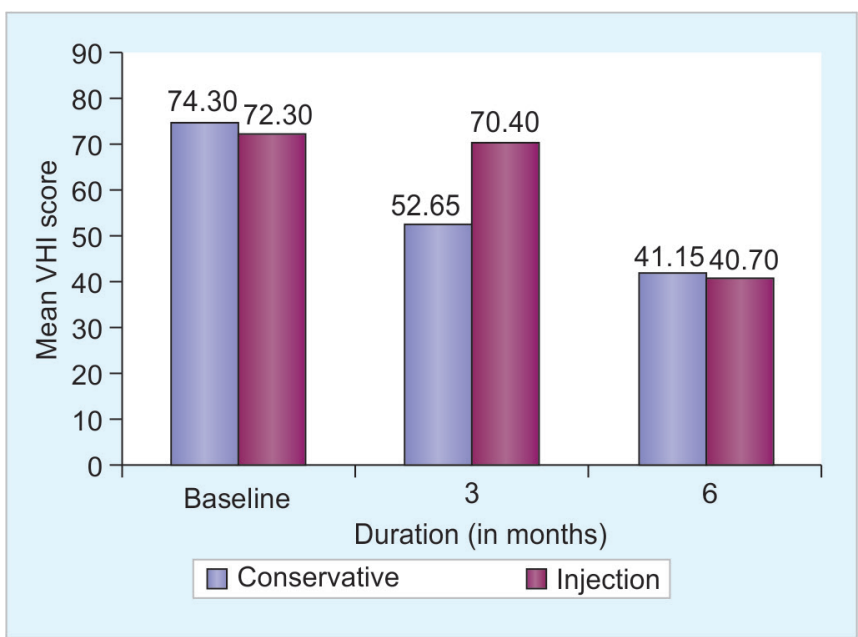

Graph 4: Comparison of changes in mean VHI score between the groups in the conservative treatment group, and $42.2 \%(\mathrm{p}<0.05)$ in the temporary medialization group from the respective values at 3 months was noted; significantly more $(p<0.05)$ in the temporary medialization group. Thus, after 6 months of treatment, a significant fall $(\mathrm{p}<0.05)$ of $44.6 \%$ in the conservative treatment group and $43.7 \%$ $(p<0.05)$ in the temporary medialization group from the baseline was observed. This was comparable between both the groups and there was no significant difference.

\section{DISCUSSION}

Unilateral vocal cord palsy is not a disease by itself but in majority of the cases it is a sign of an underlying disease or an iatrogenic injury. Dysphonia, which is the commonest symptom of unilateral cord palsy, is defined as a speech disorder resulting in impaired production of sounds by the vocal folds or any speech disorder involving problems of voice quality, pitch, or intensity under the diagnosis code 784.42 in ICD-9 (International Classification of Diseases, Ninth Revision). It has an adverse impact on a patient's social, occupational, and emotional aspects of life and leads to considerable loss of productivity from work absenteeism, especially in singers, teachers for whom the quality of voice is very important. Unilateral vocal cord palsy, if temporary, is known to recover completely by 9 to 12 months and can be aided by speech therapy and temporary medialization by injection laryngoplasty. In cases with permanent paralysis, attempts are directed at improving the voice,

Table 3: Changes in the mean VHI score between the groups

\begin{tabular}{|c|c|c|c|}
\hline \multirow[b]{2}{*}{ Duration (months) } & \multicolumn{2}{|c|}{ Mean VHI score $(\bar{X} \pm S D)$} & \multirow[b]{2}{*}{$p$-value } \\
\hline & $\begin{array}{l}\text { Conservative management } \\
(n=20)\end{array}$ & $\begin{array}{l}\text { Temporary medialization (injection } \\
\text { laryngoplasty) }(n=10)\end{array}$ & \\
\hline Baseline & $74.30 \pm 05.18$ & $72.30 \pm 04.88$ & $0.308 \mathrm{NS}$ \\
\hline 3 & $52.65 \pm 03.70$ & $70.40 \pm 04.33$ & \\
\hline 6 & $41.15 \pm 02.70$ & $40.70 \pm 13.09$ & \\
\hline Diff $(3-6 m) p$-value & $a_{-11.50 \pm 03.98(0.001)}$ & ${ }^{a}-29.70 \pm 14.72(0.001)$ & ${ }^{\mathrm{a}} 0.001$ \\
\hline Diff (Baseline $-3 \mathrm{~m}$ ) p-value & ${ }^{a}-21.65 \pm 03.39(0.001)$ & ${ }^{a}-01.90 \pm 00.88(0.001)$ & ${ }^{\mathrm{a}} 0.001$ \\
\hline Diff (Baseline $-6 \mathrm{~m}$ ) p-value & ${ }^{a}-33.15 \pm 05.37(0.001)$ & ${ }^{a}-31.60 \pm 15.09(0.001)$ & $0.755 \mathrm{NS}$ \\
\hline
\end{tabular}

Wilcoxon signed rank test (within group); SD: Standard deviation; NS: Not significant; Mann-Whitney U-test (between group); ${ }^{2}$ Significant 
preventing aspiration and decreasing the vocal fatigue. Though scientific literature is replete with data of vocal cord palsy in Western population, there is little information about the Indian scenario.

In India, Ahmed et $\mathrm{al}^{1}$ found that most patients presented in the fifth and sixth decades (77.2\%) and the most common symptom was hoarseness of voice. Pavithran and Menon ${ }^{2}$ evaluated the etiological profile of UVCP by studying a total of 121 patients in which the mean age was 46.52 years. Other international studies done by Schindler et al, ${ }^{3}$ Reiter and Brosch, ${ }^{4}$ and Algonaim et al ${ }^{5}$ have reported the mean age of their study cohort in the sixth decade of life, a decade more than our study. The difference in the age distribution between Indian and Western nations could be explained based on the higher life expectancy in the population of the affluent nations.

In our study, out of 30 patients, 19 (63.33\%) had a left-sided palsy and $11(36.67 \%)$ patients had a rightsided palsy. Similarly, left-sided preponderance was also observed in studies conducted by Yumoto et $\mathrm{al}^{6}$ from Japan, Titche, ${ }^{7}$ and Ramadhan et al. ${ }^{8}$ On the left, the RLN passes inferior and posterior to the aortic arch and reverses its course to continue superiorly into the neck, whereas the right one loops behind the right subclavian artery and ascends superomedially toward the tracheoesophageal groove. Both travel just lateral to or within the tracheoesophageal groove and enter the larynx posterior to the cricothyroid joint. Low in the neck, the course of the right recurrent nerve is more oblique, lateral, and more prone to iatrogenic injury. ${ }^{9}$ The left RLN is more frequently involved because the longer course of the nerve creates additional vulnerability, especially in the mediastinum in diseases like lung cancer, esophageal cancer, aortic aneurysm, lymphoma, tuberculosis, and mediastinal metastasis. Improved anesthetic and surgical techniques have allowed more aggressive treatment within the mediastinum, putting the nerve at further risk. ${ }^{10}$

Similar to our study, Gupta et $\mathrm{al}^{11}$ as well as Toutounchi et $\mathrm{al}^{12}$ showed that the most common symptom of unilateral vocal cord paralysis was voice change. Vocal fatigue was not much highlighted in the other published studies, whereas our study noted that this was the second most common symptom because the patients often run out of air when the vocal cords fail to approximate.

In the study carried out by Pavithran and Menon, ${ }^{2}$ the incidence of UVCP following all nonthyroidectomy surgeries together (59.3\%) was more than that of thyroidectomy $(40.7 \%)$. The most common individual surgical procedure was still thyroidectomy followed by CABG. The percentage of the patients with idiopathic palsy and with iatrogenic palsy is similar to our study. Netterville et $\mathrm{al}^{13}$ also found thyroid surgery to be the most common cause of iatrogenic RLN paralysis, thereby supporting the findings in our study.

Benninger et $\mathrm{al}^{14}$ have reported varying etiologies for UVCP and the changing trend with increasing incidence due to extralaryngeal malignancies and iatrogenic causes. A landmark study was done by Rosenthal et $\mathrm{al}^{15}$ reporting nonthyroid surgeries to be the commonest cause of UVCP. Our study does not give data on the surgery responsible for the maximum cases in nonthyroidectomy group as the data was evenly distributed. The relative incidence of surgeries causing cord palsy depends on the referral pattern in the institution, demographic parameters as well as the surgical expertise which may have contributed to the differences seen in the etiology of UVCP in our institution as compared with other published studies.

In our study, the patients failing to show satisfactory improvement at 3 months with conservative management alone were classified into the temporary medialization group, and injection laryngoplasty was done in these patients at 3 months. We used hyaluronic acid in a concentration of $22.5 \mathrm{mg} / \mathrm{mL}$. The average maintenance period of hyaluronic acid is 9 to 12 months. It is tightly cross-linked, offers excellent biocompatibility, and has the closest match to native vocal cord viscoelasticity. ${ }^{16,17}$ Hertegård et a ${ }^{18}$ demonstrated efficacy for immobile and atrophied cords that was maintained over 2 years.

Out of the 7 post thyroidectomy patients in our study, $5(71.42 \%)$ showed a favorable response to conservative management alone. Wagner and Seiler ${ }^{19}$ found that $59 \%$ of the patients had only transient paralysis postthyroidectomy, which improved over the follow-up period. Four patients of tuberculous mediastinal lymphadenopathy and one patient of pulmonary tuberculosis with apical fibrosis showed a significant voice improvement with conservative management along with antituberculous treatment. Tuberculous mediastinal lymphadenopathy causes recurrent nerve paralysis by mechanical compression over the nerve. Apical pulmonary fibrosis can cause nerve palsy by compression as well as stretching of the nerve. Rafay ${ }^{20}$ and Fowler and Hetzel ${ }^{21}$ have described cases of tuberculous lymphadenopathy of causing left RLN paralysis, which were successfully treated by antituberculosis treatment, with recovery of vocal cord function.

In our study, the patient with skull base osteomyelitis did not show significant improvement with voice therapy alone and underwent injection laryngoplasty followed by a significantly improved voice outcome. Clark et $\mathrm{al}^{22}$ and $\mathrm{Kwak}$ et $\mathrm{al}^{23}$ from Korea studied cases of skull base osteomyelitis and observed that the palsy improved with long-term antibiotics and conservative management.

Similar to the results in our study, Heuer et $\mathrm{al}^{24}$ found that after excellent voice therapy, $68 \%$ of the female 
patients and $64 \%$ of the male patients considered their voices satisfactory and elected not to have surgery, thereby stressing the importance of voice therapy. Schindler et $\mathrm{al}^{3}$ studied the vocal improvement after voice therapy in patients with unilateral vocal cord paralysis. It was found that the patients had an MPT of $5.5 \pm 2.2$ seconds before therapy and of $12.0 \pm 2.3$ seconds afterward; the difference on Student's paired t-test was significant ( $p=0.0001$ ). After therapy, GRBAS scores also showed a significant improvement. The VHI reduced from $51.4 \pm 18.8$ to $18.0 \pm 17.0$ and the difference was statistically significant $(\mathrm{p}<0.0001)$. The study concluded that the voice quality in some patients improves significantly after voice therapy alone. Mattioli et $\mathrm{al}^{25}$ from Italy also confirmed the importance of early rehabilitation with voice therapy in achieving improved voice outcomes in patients with UVCP.

Alghonaim et $\mathrm{al}^{5}$ conducted a retrospective study to evaluate the timing of injection laryngoplasty for vocal fold paralysis in an attempt to avoid future type I thyroplasty and concluded that early medialization of a paralyzed vocal fold with a temporary material appears to diminish the likelihood of requiring permanent laryngeal framework surgery. This supports our rationale in treating the patients with unsatisfactory improvement with injection laryngoplasty at 3 months. Friedman et $\mathrm{al}^{26}$ have also supported early temporary medialization. Hertegård et al ${ }^{18}$ reported that $60 \%$ of their patients had long-term benefit after hyaluronic acid injection.

In our study, injection laryngoplasty at 3 months significantly improved the voice in 8 out of the 10 patients not responding to the conservative management alone, thereby avoiding permanent medialization. Yung et $\mathrm{al}^{27}$ also concluded that the UVCP patients who underwent vocal fold injection to provide temporary medialization were statistically significantly less likely to undergo permanent medialization compared with those patients who were treated with conservative management only.

The initial treatment options for UVCP include voice therapy, observation for spontaneous return of function, or temporary medialization. Early medialization of a paralyzed vocal fold into a more favorable position can affect the final resting place of the vocal fold. This may be due to fibrosis caused by inflammation induced by the temporary agent or from reinnervation maintaining the medialized position after the injection material resorbs. If the paralysis does not resolve, permanent medialization laryngoplasty may be performed.

\section{CONCLUSION}

Unilateral vocal cord palsy occurs often due to an underlying condition or iatrogenic injury. Conservative management is the standard of care and should be started as soon as possible and given at least for 3 to 6 months. Procedure in the form of injection laryngoplasty at 3 months achieves better voice outcome in those not fully improved by the conservative management. Medialization thyroplasty should be suggested in patients who fail to show the desired improvement after temporary medialization using injection laryngoplasty.

\section{CLINICAL SIGNIFICANCE}

This study offers data on the management of patients in whom conservative approach alone for UVCP is not effective and their response to temporary medialization. The results help us to understand that an early intervention in these patients with injection laryngoplasty will prevent the requirement of permanent medialization procedures as well as give equally good outcomes compared with those benefiting with conservative management alone.

\section{REFERENCES}

1. Ahmad S, Muzamil A, Lateef M. A study of incidence and etiopathology of vocal cord paralysis. Indian J Otolaryngol Head Neck Surg 2002 Oct;54(4):294-296.

2. Pavithran J, Menon JR. Unilateral vocal cord palsy: an etiopathological study. Int J Phonosurg Laryngol 2011 JanJun;1(1):5-10.

3. Schindler A, Bottero A, Capassio P, Ginocchio D, Adorni F, Otaviani F. Vocal improvement after voice therapy in unilateral vocal fold paralysis. J Voice 2008 Jan;22(1):113-118.

4. Reiter R, Brosch S. Laryngoplasty with hyaluronic acid in patients with unilateral vocal fold paralysis. J Voice 2012 Nov;26(6):785-791

5. Alghonaim Y, Roskies M, Kost K, Young J. Evaluating the timing of injection laryngoplasty for vocal fold paralysis in an attempt to avoid future type 1 thyroplasty. J Otolaryngol Head Neck Surg 2013 Mar;42(1):24.

6. Yumoto E, Minoda R, Hyodo M, Yamagata T. Causes of recurrent laryngeal nerve paralysis. Auris Nasus Larynx 2002 Jan;29(1):41-45.

7. Titche LL. Causes of recurrent laryngeal nerve paralysis. Arch Otolaryngol 1976 May;102(5):259-261.

8. Ramadan HH, Wax MK, Avery S. Outcome and changing cause of unilateral vocal cord paralysis. Otolaryngol Head Neck Surg 1988 Feb;118(2):199-202.

9. Rubin AD, Sataloff RT. Vocal fold paresis and paralysis: what the thyroid surgeon should know. Surg Oncol Clin N Am 2008 Jan;17(1):175-196.

10. Myssiorek D. Recurrent laryngeal nerve paralysis: anatomy and etiology. Otolaryngol Clin N Am 2004 Feb;37(1):25-44.

11. Gupta J, Varshney S, Bist SS, Bhagat S. Clinico-etiological study of vocal cord paralysis. Indian J Otolaryngol Head Neck Surg 2013 Jan;65(1):16-19.

12. Toutounchi SJS, Eydi M, Golzari SEJ, Ghaffari MR, Parvizian N. Vocal cord paralysis and its etiologies: a prospective study. J Cardiovasc Thorac Res 2014 Mar;6(1):46-50.

13. NettervilleJL, Koriwchak MJ, Winkle M, Courey MS, Ossoff RH. Vocal fold paralysis following the anterior approach to the cervical spine. Ann Otol Rhinol Laryngol 1996 Feb;105(2):85-91. 
14. Benninger MS, Gillen JB, Altman JS. Changing etiology of vocal fold immobility. Laryngoscope 1998 Sep;108(9):1346-1350.

15. Rosenthal LH, Benninger MS, Deeb RH. Vocal fold immobility: a longitudinal analysis of etiology over 20 years. Laryngoscope 2007 Oct;117(10):1864-1870.

16. Hallen L, Johansson C, Laurent C. Cross-linked hyaluronan (Hyalan B gel): a new injectable remedy for treatment of vocal fold insufficiency—an animal study. Acta Otolaryngol 1999 Jan;119(1):107-111.

17. Piacquadio D, Jarcho M, Goltz R. Evaluation of hyalan b gel as a soft-tissue augmentation implant material. J Am Acad Dermatol 1997 Apr;36(4):544-549.

18. Hertegård S, Hallen L, Laurent C, Lindström E, Olofsson K, Testad P, Dahlqvist A. Cross-linked hyaluronan versus collagen for injection treatment of glottal insufficiency: 2-year follow-up. Acta Otolaryngol 2004 Dec;124(10):1208-1214.

19. Wagner HE, Seiler C. Recurrent laryngeal nerve palsy after thyroid gland surgery. Br J Surg 1994 Feb;81(2):226-228.

20. Rafay MA. Tuberculous lymphadenopathy of superior mediastinum causing vocal cord paralysis. Ann Thorac Surg 2000 Dec;70(6):2142-2143.

21. Fowler RW, Hetzel MR. Tuberculous mediastinal lymphadenopathy can cause left vocal cord paralysis. Br Med J (Clin Res Ed) 1983 May;286(6377):1562.
22. Clark MPA, Pretorius PM, Byren I, Milford CA. Central or atypical skull base osteomyelitis: diagnosis and treatment. Skull Base 2009 Jul;19(4):247-254.

23. Kwak SG, Sah DJ, Kim YJ, Kim SW. A case of central skull base osteomyelitis concurrent with multiple lower cranial nerve palsies. Korean J Otorhinolaryngol Head Neck Surg 2014;57(7):489-492.

24. Heuer RJ, Sataloff RT, Emerich K, Rulnick R, Baroody M, Spiegel JR, Durson G, Butler J. Unilateral recurrent laryngeal nerve paralysis: the importance of preoperative voice therapy. J Voice 1998 Mar;11(1):88-94.

25. Mattioli F, Menichetti M, Bergamini G, Molteni G, Alberici MP, Luppi MP, Nizzoli F, Presutti L. Results of early versus intermediate or delayed voice therapy in patients with unilateral vocal fold paralysis: our experience in 171 patients. J Voice 2015 Jul;29(4):455-458.

26. Friedman AD, Burns JA, Heaton JT, Zeitels SM. Early versus late injection medialization for unilateral vocal fold paralysis. Laryngoscope 2010 Oct;120(10):2042-2046.

27. Yung KC, Likhterov I, Courey MS. Effect of temporary vocal fold injection medialization on the rate of permanent medialization laryngoplasty in unilateral vocal fold paralysis patients. Laryngoscope 2011 Oct;121(10):2191-2194. 